\title{
A Comparative Analysis of the Adoption of Decentralized Governance in the Blockchain Through DAOs
}

\section{Youssef Faqir-Rhazoui}

Universidad Complutense de Madrid Facultad de Informatica Javier Arroyo Gallardo ( $\sim$ javier.arroyo@fdi.ucm.es )

Universidad Complutense de Madrid Facultad de Informática https://orcid.org/0000-0001-6127-7538

\section{Samer Hassan}

Universidad Complutense de Madrid Facultad de Informatica

\section{Research}

Keywords: Blockchain, DAO, Ethereum, xDai, Governance, voting system

Posted Date: February 4th, 2021

DOI: https://doi.org/10.21203/rs.3.rs-166470/v1

License: (c) (1) This work is licensed under a Creative Commons Attribution 4.0 International License.

Read Full License 


\section{RESEARCH}

\section{A Comparative Analysis of the Adoption of Decentralized Governance in the Blockchain Through DAOs}

\author{
Youssef Faqir-Rhazoui ${ }^{1}$ \\ , Javier Arroyo ${ }^{1,2^{*}}$ \\ and Samer Hassan ${ }^{1,2,3}$ \\ ${ }^{*}$ Correspondence: \\ javier.arroyo@fdi.ucm.es \\ 2 Institute of Knowledge \\ Technology, Universidad \\ Complutense de Madrid, Spain \\ Full list of author information is \\ available at the end of the article
}

\begin{abstract}
Blockchain technology has prompted new ways of governance and coordination. Such forms receive the name of Decentralized Autonomous Organizations (DAOs). DAOs typically implement decision-making systems to make possible for the community to reach agreements. As a result of the agreements the DAO automatically operates (e.g., hire people, payments, invest in financial products, etc.) by executing code on the blockchain.

In the last few years, several platforms such as Aragon, DAOstack and DAOhaus, have emerged to facilitate the creation of DAOs. As a result, hundreds of this new organizations have appeared and are interacting in the blockchain. However, little is known about them. In this paper, we want to shed light on them. We will review the three main platforms (Aragon, DAOstack, DAOhaus) that make possible to create and manage a DAO. We introduce the main differences between these platforms, and we finally compare them numerically. For the comparison we retrieve data from both the main Ethereum network (mainnet) and a parallel Ethereum network (xDai). We analyze data from 72,320 users from 2,353 DAO communities to study the three ecosystems along four dimensions: growth, activity, voting system and funds. Our results show that there are notable differences among the DAO platforms in terms of growth and activity, but also in terms of voting results. Still we consider that our work is only a first step and that further research is needed to better understand these communities and to help them to accomplish their aim of decentralized governance.
\end{abstract}

Keywords: Blockchain; DAO; Ethereum; xDai; Governance; voting system

\section{Introduction}

Public blockchains provide a mechanism in which decentralized transactions and operations are secure, without needing to trust a third-party as in centralized systems $[1,2]$. While blockchain technology is mostly used to run cryptocurrencies and financial products, other applications can benefit from the capabilities of this new technology. In particular, new forms of decentralized governance have emerged in the blockchain. This new organizations are known as Decentralized Autonomous Organizations (DAOs) and provide a way of interaction with features such as transparent decision processes, autonomous automation, serverless infrastructure, etc [3].

So far the blockchain has attracted mainly experts and enthusiasts about computing technology and also to fintech advocates that are looking for ways to take 
advantage of the blockchain technology to create new financial services. Some of these people organize through DAOs to govern their projects. DAOs have decisionmaking mechanisms embedded and in this way the community govern the DAO by means of voting proposals for example to do tasks for the organization. In the last few years, several platforms have appeared to provide DAO deployment and interaction as-a-service and in this way reducing the technical knowledge that a group of people need to operate through a DAO. As a result, thousands of people are now interacting through thousands of DAOs.

This new phenomenon can be followed on the Internet, particularly, through 'grey literature' including technical reports, blogs, social media posts, etc. However, very recently it has started to attract research attention. One of the first attempts can be found in [4], where the authors analyze in a systematic way the grey literature on three popular DAOs to understand how are they governed. In [5], the authors offer an overview of DAOs, DAO platforms and DAO visualization tools, and analyze the evolution of one popular DAO using quantitative data. In [6], three of the main DAO platforms are analyzed in terms on their functionalities for building DAOs. As it can be seen, research literature

In this paper, we will contribute to the growing stream on literature on the topic by providing statistics of the three of the main DAO platforms (Aragon, DAOstack and DAOhaus) in terms of growth, activity, voting system and funds. This will help to understand the adoption

The rest of the article proceeds as follows. Section 2 introduces the main concepts of blockchain and Ethereum and Section 3 follows explaining what a DAO is. In Section 4, we review the three DAO platforms that we are going to analyze in this work. Section 5 compares the three main DAO platforms in terms of growth, activity, voting system and funds. Finally, Section 6 concludes with a discussion on the main findings and the limitations of our work.

\section{Blockchain: The field in a nutshell}

Blockchain is a distributed ledger, which can be thought as a distributed appendonly database with a synchronization mechanism. Like the Internet, the public blockchain is an open infrastructure, not owned or controlled by one central authority. Generally, the ledger is copied in each of the network nodes, and thus can be viewed by all its users $[7,2]$.

The ledger is a sequence of blocks (hence block-chain) that contains a set of transactions already performed. ${ }^{[1]}$ Each block points to the previous block in the ledger, forming a chain.

When a user wants to add a new transaction to the ledger, the transaction data is verified by the so-called miners. If there is consensus on the new block validity, it is added to the chain in a decentralized process $[1,2]$. Furthermore, the blockchain grants immutability of its past records: nobody can delete and alter the data of the block placed [8].

${ }^{[1]}$ In cryptocurrencies, each block holds transactions, i.e. movements of cryptocurrency between accounts. In other more general applications such as Ethereum-based apps (and DAOs), blocks contain operations, akin to typical instructions in a computer program, that need to be executed. 
The first implementation of the blockchain technology was Bitcoin, which is a "crypto-currency", i.e. decentralized digital currency validated through cryptography [9]. After that, thousands of new cryptocurrencies have emerged with their own features [10].

The second wave of blockchain was prompted by the advent of Ethereum in 2013 [11]. Ethereum provides a distributed computing platform and a programming language, Solidity [12]. Solidity addressed several limitations of the Bitcoin's scripting language, like the lack of Turing-completeness [13]. This has enabled multiple types of decentralised applications (Dapps) and the so called "smart contracts", computational agreements between parties which may be self-executed and self-enforced.

Dapps have been applied in many fields $[14,15]$. One of the most important ones is economy, include financial applications such as general banking services [16] or cryptocurrency payments [17], leading to the surge of Decentralized Finance (DeFi), a form of finance that does not rely on central financial intermediaries use to get crypto-savings, crypto-loans, or even trade with them [18]. Other fields, like IoT, are using blockchain as a common communication layer [19].

Ethereum and smart contracts also enable new forms of decentralized governance such as Decentralized Autonomous Organizations (DAOs), that are organizations where decision-making is distributed or delegated away from a central authority.

\section{Decentralized Autonomous Organization}

A DAO is a blockchain-based system that enables people to coordinate and selfgovern themselves mediated by a set of self-executing rules deployed on a public blockchain, and whose governance is decentralized, that is, independent from central control [20].

Commonly, a DAO is formed by a group of agents which are identified by a unique address. In some cases, the way that these agents join a DAO, is by investing in it to get an amount of the DAO participation (named tokens), which is exactly as a corporation does.

DAOs are considered autonomous because their work is enabled and self-enforced by a set of rules, which are in simple terms, code that runs in the blockchain (e.g., hire people, buy/sell resources for/to the community). Therefore, this virtual entity is totally independent of its creators and cannot be influenced from the outside.

Regarding decentralization, it comes from the fact that all the decisions (and consequently the actions) a DAO takes, are taken through by consensus between DAO members. This consensus is usually reached by voting, where all the DAO members can participate.

Notice that those decisions do not just concern the allocation of the DAO resources (e.g., funding projects, payments) but also could change the DAO code. For example, even if a bug in the code of the smart contract comes out out, it would require a vote to change the code [21].

The notion of DAO is tied to the blockchain $[22,23]$. The technology behind the implementation strongly characterizes the resulting organizations. The DAO activity ${ }^{[2]}$ is recorded in the blockchain and, as a result, it implies a cost. Validating and confirming transactions on the Ethereum blockchain requires a certain amount

${ }^{[2]}$ Those actions basically are transactions on the blockchain. 
of work and computational resources, called gas and paid in cryptocurrency. This work is performed by blockchain miners in order to include transactions in a block. Gas ultimately translates into money and the amount of gas depends on the size and type of each transaction. Hence, the blockchain can be seen as a costly and secure database system. This conditions the amount of information that is stored in the blockchain and, consequently, will affect the behavior of the communities that operate in the blockchain. A significant example is that DAO members use other means for communication including forums such as DAOtalk. ${ }^{[3]}$

While Bitcoin can be considered a proto-DAO $[24,25]$ it is probably not "smart" enough because it is not an entity by itself and can not autonomously do things (except the mining protocol). One of the first DAO implementations was The DAO, launched in April 2016 in the Ethereum blockchain by a group of programmers. It was the most successful investment crowdfunding at that time. The DAO was a sort of hedge fund, in which contributors could directly vote proposed projects. In June 2016, due to an error in The DAO code, an attacker robbed a large part of its funds [26]. The impact of the event made the Ethereum Foundation to hard fork the project ${ }^{[4]}$ and returned the stolen funds to The DAO investors. However, the concept of immutability of the ledger past records was damaged due to the fork [27].

Despite that traumatic event, the endeavor of creating decentralized organizations to operate in the blockchain persisted. As creating a DAO from scratch requires highly specialized knowledge about blockchain programming, new solutions have emerged that provide templates that enormously reduce the technical knowledge required to deploy a DAO in the blockchain.

\section{DAOs enabled by a platform}

The platforms that provide DAO deployment as-a-service allow users with scarce knowledge on how blockchain works to create a DAO using a template that typically can be customized. The main platforms are Aragon, DAOstack, DAOhaus and Colony [5].

However, Colony will not be covered in this article due to the lack of data availability. We will mention that the way that a Colony DAO works breaks with the "traditional" vote-driven schema of functioning, where each action of the DAO must be voted. In Colony, DAOs are work-driven, which means that works are published, and members accept them for a payout [28]. So, comparing vote-driven and work-driven DAOs makes no sense due to the different nature of their conceptions.

\subsection{Aragon}

Aragon ${ }^{[5]}$ is by far the largest DAO platform. Aragon aims to extend the use of DAOs as a free and open-source technology to allow the creation and management

[3] https://daotalk.org/

${ }^{[4]}$ A "hard fork" means the blockchain is copied in a new version with some difference (e.g. different rules or some blocks removed). This provokes a divergence in the two paths forward the blockchain can take. Typically, only one is considered the "valid" path; however, both can still be used.

${ }^{[5]}$ https://aragon.org/ 
of decentralised organizations [29] under different forms including companies, cooperatives, nonprofits, or open-source projects. ${ }^{[6]}$

Aragon provides a static template to make your own DAO, but it also allows you to create a customized one. Customization is enabled through "apps" (sets of smart contracts), which can be installed or removed from DAOs by a voting. The purpose of apps widely varies: a Finance app to allocate the DAO's funds; an Agent app to interact with other Ethereum smart contracts; a Token app to manage the membership; or a Vote app used as a decision-making system [30, 31]. In addition, Aragon provides a $S D K^{[7]}$ to create and deploy their smart contracts, apps, and organizations templates (i.e. a set of predefined apps and a customized configuration for the template purpose).

In this article, we will focus on the Vote app, because voting is the main action in most DAOs. Furthermore, the default Aragon Voting app is the most used in Aragon DAOs, and its decision-making system work as follows. The app defines two conditions that any voting must fulfill to be approved.

1 From all casted votes, the percentage of positive casts in casted votes must be greater than or equals to the support required percentage.

2 The minimum acceptance quorum parameter states the minimum percentage of votes casted from all possible votes in the DAO.

The first one establishes the majority acceptance from the casted votes, while the second one establishes the minimum participation required. Both parameters can be changed by voting.

There are other apps for voting. For example, there is an in-development app which implements the decision-making system of the DAOstack platform [32], or the Dandelion voting app which implements the decision-making system of Moloch implemented in the DAOhaus platform, both will be covered below. Furthermore, the Dot-Voting app adds the possibility to vote with more than two answers instead of the typically binary answer (yes/no).

Even you can create a new organization template to change, not just the voting app, but the organization sort; for example the Committee template [33, 34]. This template was created to face the scalability problem (see section 4.2). ${ }^{[8]}$ For that, they propose to break the DAO group into specialized sub-groups in charge of specific tasks, which can autonomously work without the interaction with the whole group.

Another ambitious decision-making systems implemented as an Aragon app is Conviction Voting (CV) or Social Sensor Fusion [35]. In CV, DAO members cast votes, with an amount of the DAO token they have, in a proposal. For example, member $A$ has fifty tokens, and she votes with twenty tokens in proposal $A$. So, now member $A$ has just thirty tokens to vote in other proposals. Hence, if she wants to use more tokens than the remaining, she will have to remove tokens from proposal $A$. That is the conviction. The second key of $\mathrm{CV}$ is that proposals have to pass a dynamic threshold to be approved. This threshold is tied to the treasury funds. In

\footnotetext{
${ }^{[6]}$ https://help.aragon.org/article/4-about-aragon

${ }^{[7]}$ https://hack . aragon.org/

${ }^{[8]}$ Scalability in terms of growing a DAO membership and its operations (i.e., votes, tasks, etc.)
} 
other words, the more budget the proposal requests, the more conviction (tokens) the proposal has to gather. Notice that if proposal $B$ is approved before proposal $A$, then the DAO funds decrease, and the proposal $A$ threshold will increase. On the other side, if the DAO funds increase, the threshold will decrease [36, 37]. Conviction Voting was tested through simulations, ${ }^{[9]}$ and Aragon makes possible to deploy it in a real environment [38].

\subsection{DAOstack}

DAOstack ${ }^{[10]}$ is a platform that aims to tackle the governance scalability problem. Matan Field, co-founder of DAOstack, states that the bigger a DAO is, the harder it is to manage it [39]. Consider a DAO where all the decisions are taken by voting, in a DAO with few members, the number of decisions will be bound to the number of members, and a majority voting to approve those decisions will involve a sub-set of those few users. However, if we increase the number of those users, then the number of decisions and subsequently the number of users needed to approve them will also increase. That is important because it always requires enough mindful members to approve decisions. A naive solution to this could be reducing the required quorum (i.e., a relative majority), but it also introduces new flaws. For example, an attacker could spam lots of decisions in a small frame-time requesting the DAO funds, and it will be easier to get the funds using a lower quorum. Matan Field states that increasing the DAO membership will reduce its resilience [39].

To face this problem, DAOstack proposes the Holographic Consensus (HC) decision-making system [40, 41]. In HC, DAO members send and vote for proposals. Proposals are approved by absolute majority. And finally, the stakes, in other words, people $^{[11]}$ stake their (Gen) tokens trying to guess if a proposal will be approved. If it finally is, the staker will earn a reward, if not they will lose the tokens staked. If a proposal receives enough stakes, it will not require an absolute majority voting, but a relative one. If staking works properly, which apparently it does [42], it acts as a filtering mechanism that highlights the most relevant proposals specially in larger DAOs where absolute majorities demand too much attention from the community. With HC, DAO members do not spend that much time deciding what proposals are really interesting for the community because stakers actually filter the good proposals from the bad ones. To be rewarded, stakers need to be aligned with the DAO global opinion; otherwise, they will lose their investment.

\subsection{DAOhaus}

DAOhaus ${ }^{[2]}$ is the platform where to create DAOs that work in the same way as Moloch DAO. Moloch DAO was a grassroots answer to coordination problems in funding Ethereum 2 and other community grants.

DAOhaus DAOs implement a straightforward voting system, which is basically a non-quorum system, where always a relative majority is enough to approve a proposal. This way to proceed simplifies development and testing processes [43] of

\footnotetext{
${ }^{[9]}$ https://github.com/1Hive/conviction-voting-cadcad

${ }^{[10]}$ https://alchemy. daostack.io/

${ }^{[11]}$ They can be DAO members or people from outside the DAO.

${ }^{[12]}$ https://daohaus. club/
} 
their voting system. A key aspect from these DAOs is the "rage quit" mechanism that makes possible to exit a DAO with your portion of the DAO resources if you do not agree with the result of a voting. After the voting outcome is achieved, there is a 'grace' period, when DAO members can quit if they are not agree with the outcome. Additionally, if there are more than $\approx 30 \%$ of rage quits, then the vote will be automatically rejected [44].

In addition, this voting system has two main attributes to consider: shares and tributes. Shares refer to an amount of resources that each DAO member has, independently of the cryptocurrencies the DAO has. And tributes refer to an amount of shares the proposal applicant pays to the DAO. Thus, in a proposal, the applicant can request shares and pay a tribute. Either shares or tribute defines the proposal sort, e.g. the same amount of shares and tribute defines a membership proposal, only share request defines a project proposal, and only tribute payment means a donation to the DAO [45]. If the proposal sent fails, then the applicant will get back her tribute offering.

However, Moloch v2 has introduced a new element, the sponsorship, which slightly changes the voting system. Now, when a proposal is sent, it requires the sponsorship of a DAO member. This means that any DAO member makes a deposit confirming that it is trustful. All the proposals need to be sponsored before moving them to the regular queue, where the voting starts [44]. When the voting ends, independently of the outcome, the sponsor will get a portion of her deposit back. In this way they intend to avoid attackers to spam lots of proposals to exploit the non-quorum characteristic of Moloch voting system.

Nowadays, DAOhaus DAOs are split into two groups. Those created in the early stage of DAOhaus, v1 DAOs, and those created with new features of Moloch, v2 DAOs. Some changes introduced from v1 to v2 DAOs, include the ability to expel a DAO member from the community. It also includes some changes like the ability to send proposals by non-DAO members or the changes related to its voting system [44] already mentioned.

\section{Quantitative comparison of the three main DAO platforms}

We will compare the three DAO platforms introduced in the previous section, i.e., Aragon, DAOstack, and DAOhaus, using two DAO visualization tools: DAOAnalyzer ${ }^{[13]}$ for the adoption and activity metrics, and DeepDAO${ }^{[14]}$ for the funds statistics. The data used in this comparison covers until November 30th. The comparison will tackle four topics; growth, activity, voting system, and the funds owned by DAOs.

In our comparison, we will include both the DAOs deployed in the Ethereum mainnet and the DAOs deployed in the $x$ Dai network. As already explained the use of the Ethereum mainnet implies the payment of a fee (e.g., gas cost), and this fee is tied to the network's use. In mid-2020, the Ethereum mainnet was overused, so the fee to process a any transaction (e.g., vote, create a DAO, etc.) was extremely high. As a result, some alternatives emerged to avoid such costly prices. That was the case of the $x D a i$ network that is a blockchain (a sidechain indeed) designed for

\footnotetext{
${ }^{[13]}$ http://dao-analyzer-science

${ }^{[14]}$ http: //deepdao. world
} 
fast and inexpensive transactions. It has a bridge with mainnet to bring tokens or to give them back. As it can be seen in Table $1,{ }^{[15]}$ which shows the costs to create a DAO (summon), and to vote in the DAOhaus ecosystem, Ethereum mainnet was much more expensive and slow than $x$ Dai, which in turn is not as secure as mainnet.

\begin{tabular}{|l|l|l|l|}
\hline & Summon & Vote & Speed \\
\hline mainnet & $\$ 80$ & $\$ 5$ & 5 tps \\
\hline xDai & $\$ 0.01$ & $\$ 0.001$ & 70 tps \\
\hline
\end{tabular}

Table 1: Comparison of mainnet and xDAI networks in DAOhaus.

\begin{tabular}{|c|c|c|c|c|c|c|}
\cline { 2 - 7 } \multicolumn{1}{c|}{} & \multicolumn{2}{c|}{ Aragon } & \multicolumn{2}{c|}{ DAOhaus } & \multicolumn{2}{c|}{ DAOstack } \\
\hline & mainnet & $\times$ Dai & mainnet & xDai & mainnet & xDai \\
\hline DAOs & 1,744 & 325 & 169 & 56 & 22 & 37 \\
\hline Users & 41,021 & 17,660 & 1,180 & 265 & 6,645 & 5,549 \\
\hline Proposals & 10,246 & - & 1,668 & 554 & 1,954 & 463 \\
\hline
\end{tabular}

Table 2: Comparison of the three ecosystems in terms of number of DAOs, users and proposals

Table 2 shows the number of DAOs, users, and proposals per platform and by network (mainnet and xDai). Aragon is by far the most important platform in terms of DAOs, users and proposals. Regarding the number of DAOs, DAOhaus comes second with more than 200 DAOs in mainnet and xDai, while DAOstack has 59. However, in terms of users DAOstack is more populated than DAOhaus.

Regarding the $x D a i$ adoption, in Aragon $x D a i$ DAOs represents $15.71 \%$ of the total number of DAOs, while $x$ Dai users are $30.1 \%$ of the total. Aragon started using $x$ Dai from July 2020. There is no data available from proposals.

In the case of DAOhaus, $x$ Dai DAOs are $24.89 \%$ from its total, while $x$ Dai users are $18.34 \%$, and the proposals represent $24.93 \%$ of all proposals. As in the case of Aragon, DAOhaus uses xDai since July 2020.

Finally, in DAOstack the DAOs in $x$ Dai are $62.71 \%$ from its total, $x$ Dai users are $45.51 \%$, and the number of proposals are $19.16 \%$ from the total. The adoption in terms of users and DAOs is significantly higher than in the other two platforms. This may be explained by the fact that DAOstack started using $x$ Dai since February 2020.

Due to the importance of the $x D a i$, we will include it in the comparisons in the following sections.

\subsection{Growth over time}

For the growth comparison, we will use two metrics: the number of DAOs and the number of users. However, the timestamp of the DAO creation currently is not available for DAOstack DAOs, while the timestamp of the user registration is not available for Aragon DAOs.

Figure 1 shows that the growth of Aragon in mainnet was constant and it was boosted by $x$ Dai. On the other hand, the growth exhibited by DAOhaus was more modest. The new DAOs created in $x$ Dai can be brand new DAOs or DAOs that 'migrated' from mainnet DAOs; however, the DAO migration implies the creation of a new DAO with a different id and account. So there is no way for us to detect when a $x$ Dai DAO is new or the result of a migration process.

[15] Source: https://daohaus.club/help\#xDAI 


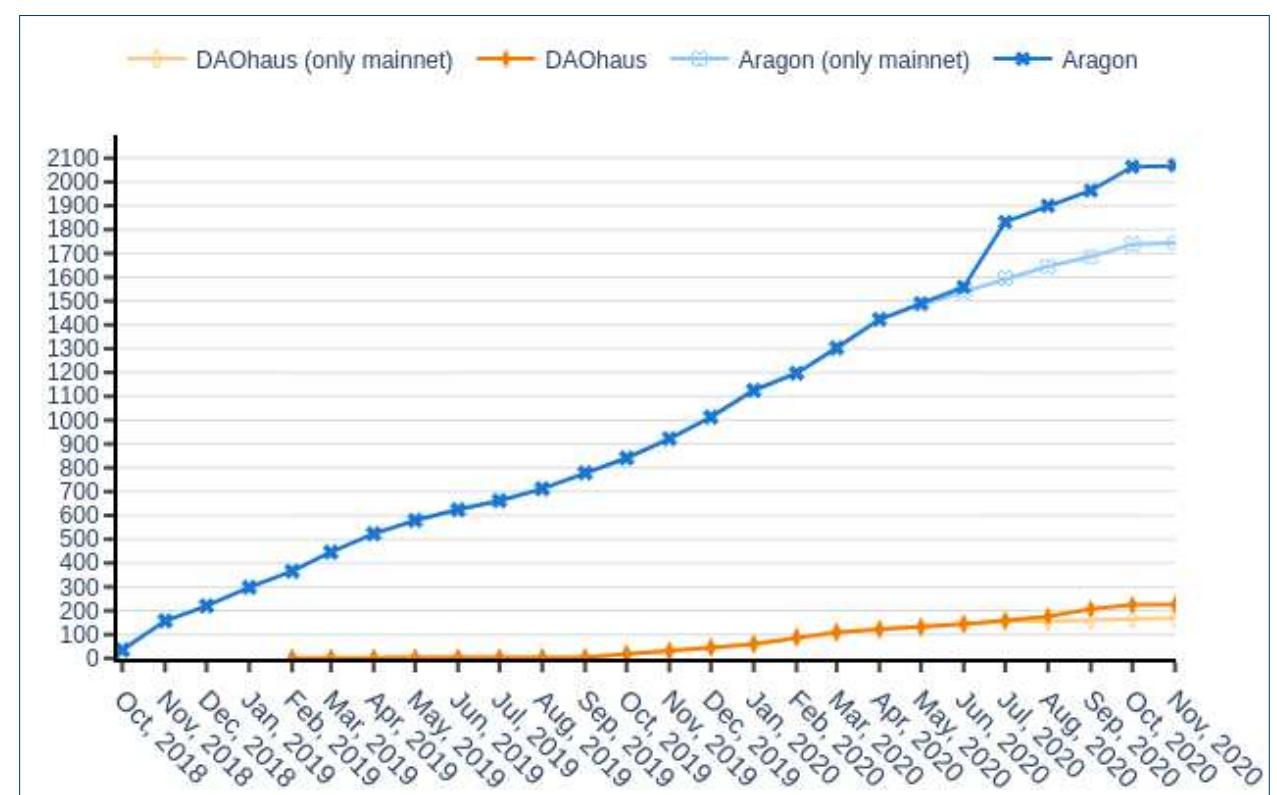

Figure 1: Evolution of the number of DAOs of Aragon and DAOhaus.

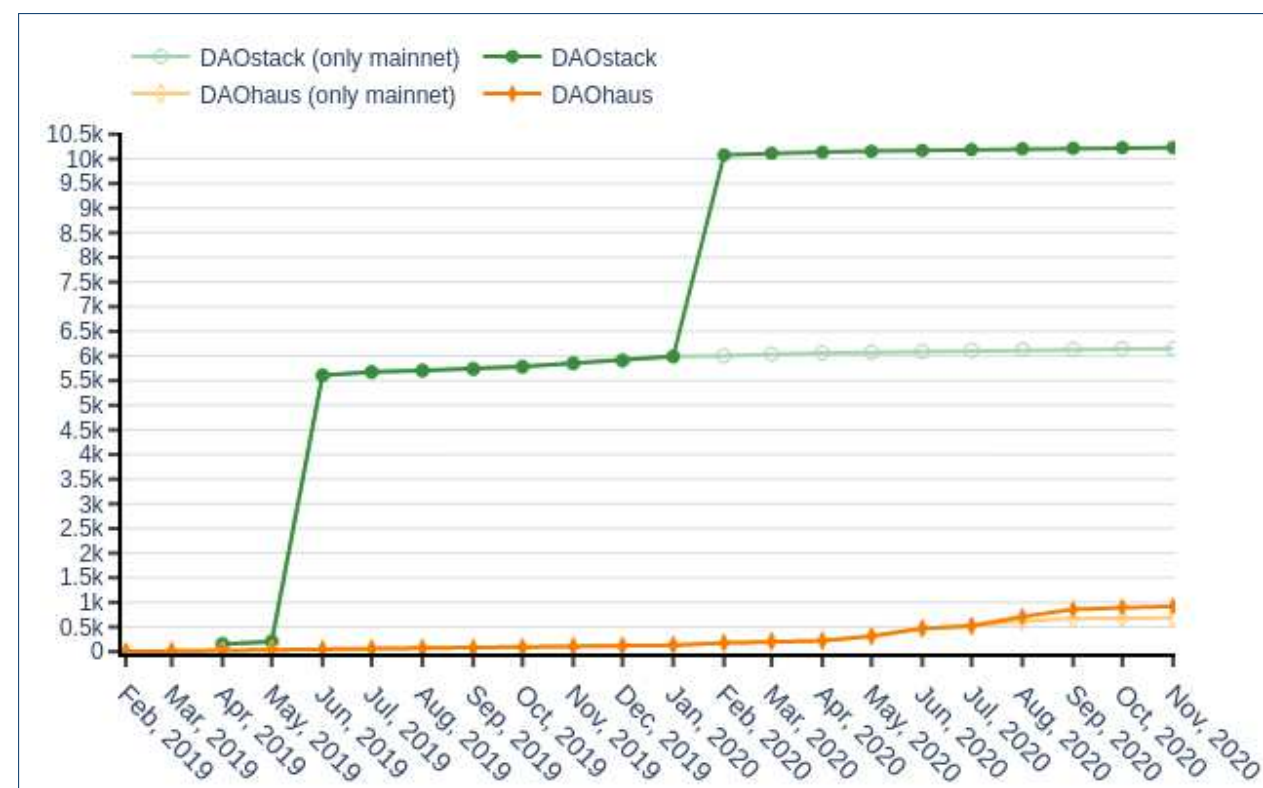

Figure 2: Evolution of the number of users in Aragon and DAOhaus.

Figure 2 shows the number of users of DAOhaus and DAOstack. We can see two steps in the DAOstack series. The first step took place in June 2019 when 5,397 new users joined the project and almost 5,000 joined to the same DAO [42]. In February 2020, the second step $(2,822$ new users) was due to the launchment of $x$ Dai network (the gap between the dark and light green lines). Besides these two steps, the increase was steadier and much more modest.

By contrast, the users growth in DAOhaus had no significant increases. Still, the growth is more pronounced since April 2020. In addition, as we previously explained 
in Section 4.3, DAOhaus users can quit from a DAO (rage-quitting). Thus, the number of users would be higher, because 311 people used the 'rage quit' option during the period analyzed and such option is not available in other platforms, where users just abandon their accounts.

\subsection{Activity over time}

First, we need to define what means 'active' for both a DAO and a user. We will follow the definition in [5] that consider that a DAO or a user were active in a given month if at least they performed an action in that month. The actions depend on the platform. For DAOstack we will consider as actions: create a proposal, vote a proposal, and stake in a proposal. In the case of DAOhaus: create a proposal, vote a proposal, and quit the DAO. Finally, due to the customization of Aragon DAOs, it is difficult to homogenize the actions because of the multiple possible apps to install. So, in the case of Aragon we will just consider data from the basic voting app (create a proposal, known as vote in Aragon, and cast a vote), and from the transaction app, used for donations or payments, where we will consider transactions as actions. However, for Aragon xDai actions, we can only consider data from the transaction app because the API does not provide data from the voting app in $x D a i$.

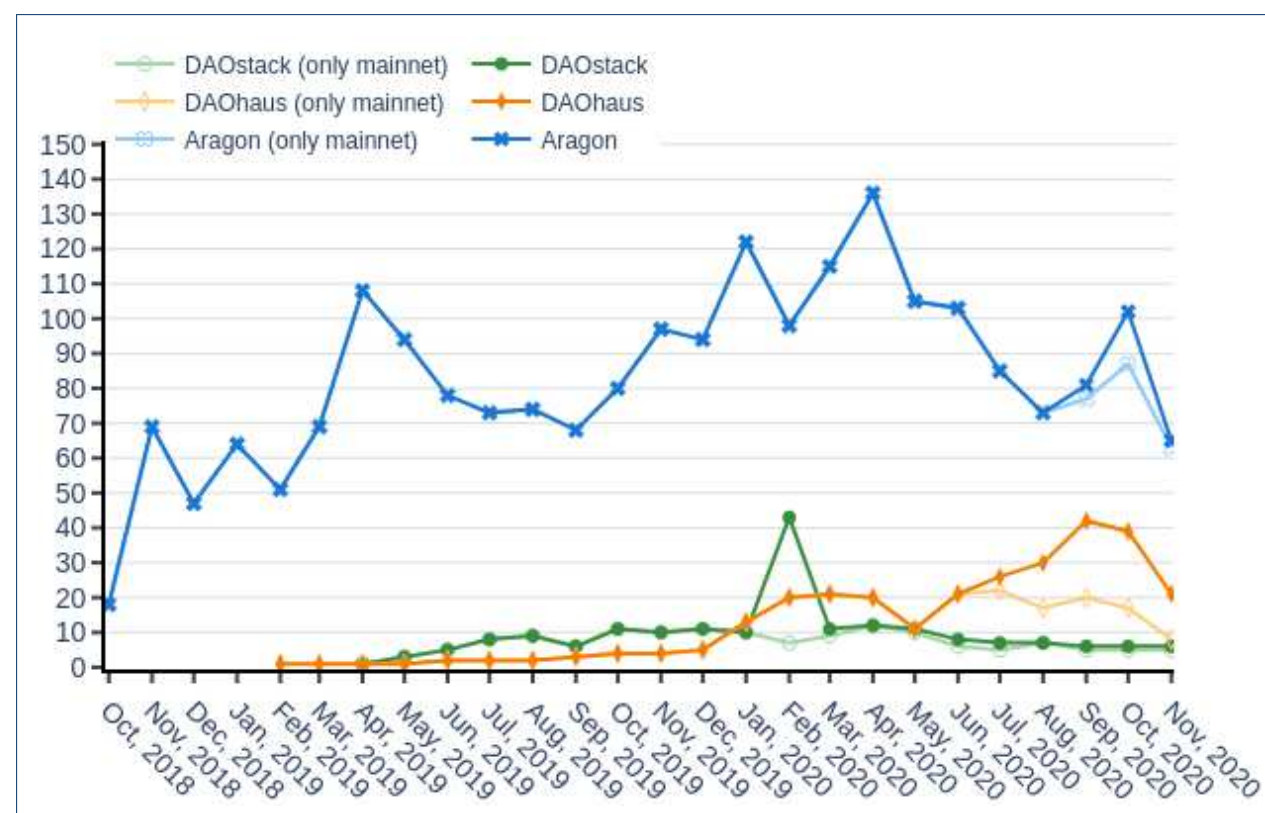

Figure 3: Number of active DAOs in Aragon, DAOhaus and DAOstack.

Figure 3 shows that Aragon has the highest number of active DAOs in Aragon, even considering only transactions, so the number of active DAOs should be higher. Still the number seems small (around 100) considering the number of DAOs registered (over 2,000), we can also observe a negative trend since May 2020.

DAOhaus apparently follows an increasing trend that also has benefited from $x$ Dai network, greatly increasing its active DAOs to forty per month. Finally, the number of active DAOs in DAOstack is more modest (around 10) and $x$ Dai did not suppose an increase, but it seems to remain stable during the last year and a half except for an activity surge in February 2020 due to the xDai adoption. 


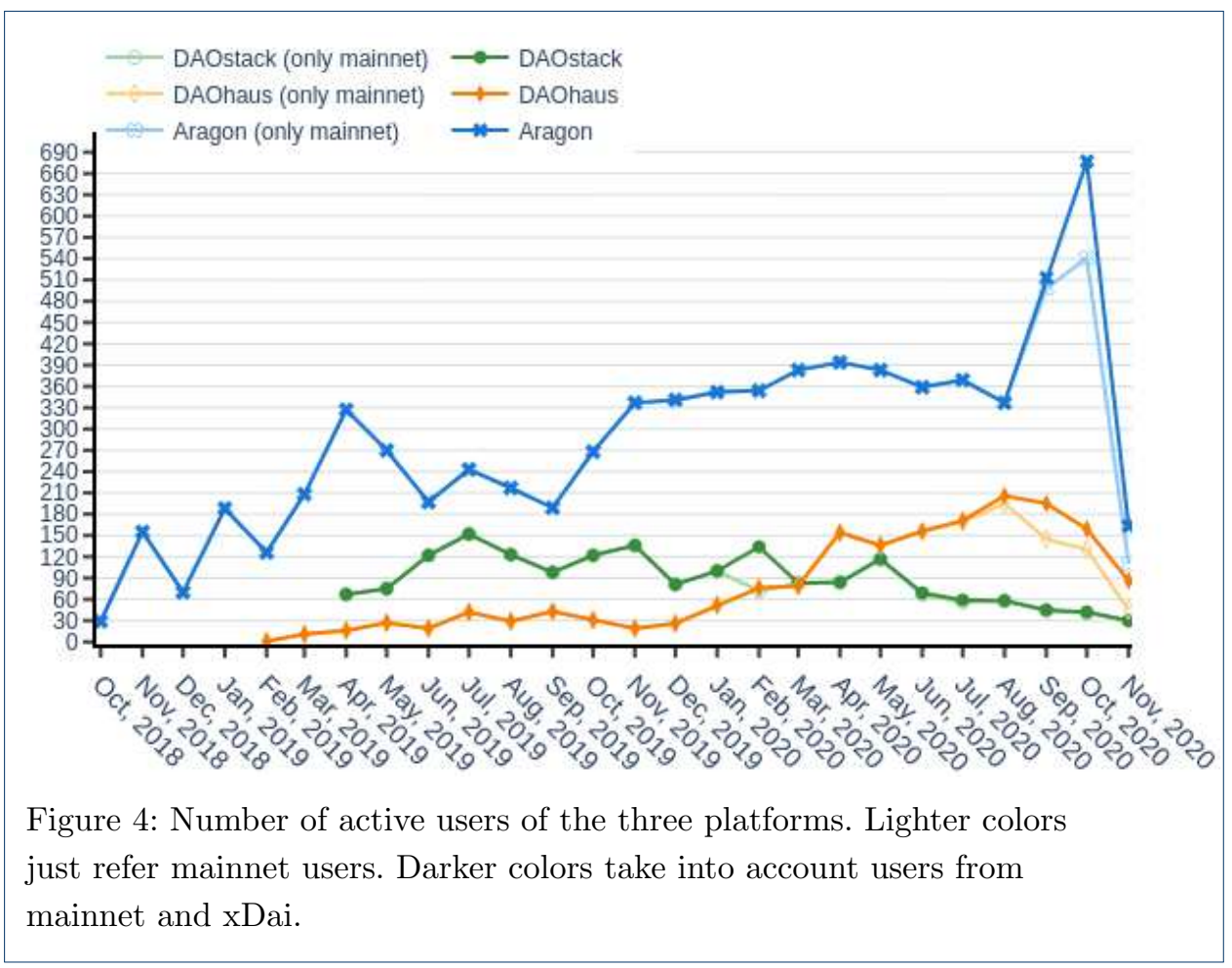

Figure 4 shows the activity in terms of users. Again Aragon has higher number than the other two platforms. The number of users is increasing, but the last three months show an volatile behavior.

While DAOhaus has been continuously increasing the number of active users, DAOstack has been decreasing since June 2019. According to this metric, the impact of $x$ Dai has not boosted the activity of DAO users.

\subsection{Voting system}

The three platforms considered are vote-driven, but each of them have their own voting system, as we explained in Section 4. In the case of Aragon, DAOs, they can have many voting systems, but, for the sake of simplicity, we just retrieved data from the standard voting app.

In order to compare these decision-making systems, we have chosen three metrics:

- The percentage of users who vote, which can help us to see the engagement of the community with their DAO.

- The number of casted votes per voter, which will show us how active voters are in terms of participation

- The percentage of proposals that are approved, which can show how the voting system could influence the results.

As we did for the previous metrics, we will also consider the mainnet, xDai network, and the sum of them.

Table 3 shows the statistics of voting for the three platforms. Interestingly, both in DAOstack and Aragon the percentage of users that vote is less than $10 \%$, while in DAOhaus is close to $40 \%$. In the case of DAOstack the cause can be the inactivity of the DAO with around 4,000 users, while in the case of Aragon the high number of 


\begin{tabular}{|l|l|l|l|l|l|l|l|l|l|l|}
\cline { 2 - 10 } \multicolumn{1}{c|}{} & \multicolumn{3}{c|}{ DAOstack } & \multicolumn{2}{l|}{ DAOhaus } & \multicolumn{2}{l|}{ Aragon } \\
\cline { 2 - 10 } \multicolumn{1}{c|}{} & total & mainnet & xDai & total & mainnet & xDai & total & mainnet & xDai \\
\hline Users who vote & $4.5 \%$ & $6.3 \%$ & $2.1 \%$ & $38.37 \%$ & $39.5 \%$ & $24.32 \%$ & - & $6.18 \%$ & - \\
\hline Votes/voter & 4.6 & 4.64 & 3.64 & 4.26 & 3.96 & 7.28 & - & 4.08 & - \\
\hline Approved prop. & $74 \%$ & $74 \%$ & $76 \%$ & $92 \%$ & $93 \%$ & $87 \%$ & - & $81 \%$ & - \\
\hline Votes-for & $86 \%$ & $86 \%$ & $95 \%$ & $91 \%$ & $90 \%$ & $98 \%$ & - & $94 \%$ & - \\
\hline
\end{tabular}

Table 3: Voting statistics by platform and network

inactive DAOs. Surprisingly the percentage of users who vote in $\mathrm{xDai}$ is smaller than in mainnet. A potential explanation could be that $\mathrm{xDai}$ is a younger alternative, specially in DAOhaus.

Regarding the votes per voter ratio, in all the platforms the ratio is around 4 . The main difference that we observe is that in DAOhaus the ratio in xDai almost doubles that in mainnet, which could mean that xDai boosted participation, but according to Figure 4, it does not come from more active users, because the number of active users in xDai decreased since the beginning of its adoption; hence, it should be an activity increase in the users that are active in xDai.

Regarding the percentages of approved proposals, the values are high for all the platforms. It might be due to the fact that DAO members mainly present proposals that they believe that can be approved, because they are generally aligned with the rest of the community and because the cost (in gas, reputation and time) of presenting proposals that are likely to be rejected. However, still there are important differences in the percentage of approved proposals across platforms. DAOstack has the lowest values (around 75\%), followed by Aragon (81\%) and then DAOhaus (around 90\%).

In the case of DAOstack voting system, it might be explained because it requires an absolute majority by default, or enough staking for a proposal to can be approved by relative majority. So non-boosted proposals are more likely to be rejected. According to the analysis in [42], such thing mainly happens in bigger DAOs (those with more than 23 members), which are those that need holographic consensus to facilitate the approval of a proposal.

The high number of approved proposals in DAOhaus can be explained because the voting system requires no quorum, which is a filter to reject proposals that have no sufficient support, and also because the proposals v2 DAOs also require the sponsorship, which acts as a preliminary filter of bad proposals.

In the case of Aragon, we find a percentage of approved proposals between DAOstack and DAOhaus. Since the Aragon standard voting app requires a quorum to approve a proposal, it makes sense that the approval rate are below to that from DAOhaus. The aggregated results show that the Aragon voting system with the personalized configurations lead to lower rejection rates than the DAOstack one, that in some cases requires a majority voting to pass a proposal.

Please, notice that we are not stating that a voting system is more effective than the others, we are just interpreting the influence of those decision-making systems on the general figures. Still our conclusions should be validated through further studies. 


\subsection{Funds}

Funds are an important aspect in DAOs to cover, because generally DAOs employ people or fund proposals that can benefit the community (i.e. physical meetings, hackathons), or for investing, etc. We will study the DAO funding in terms of the main cryptocurrencies. For that purpose, we have used DeepDAO which apparently does not provide information from all the DAOs in the considered ecosystems, but from many of them, including the most important ones.

\begin{tabular}{|c|r|r|r|}
\hline Token name & Token symbol & DAOs which use it & Total USD which DAOs have \\
\hline Dai stablecoin & DAI & 51 & $6,229,754 \$$ \\
\hline Ether & ETH & 50 & $14,714,446 \$$ \\
\hline Sai stablecoin v1.0 & SAI & 21 & $15,013 \$$ \\
\hline USD Coin & USDC & 20 & $5,878,148 \$$ \\
\hline Wrapped Ether & WETH & 18 & $9,303,476 \$$ \\
\hline Aragon & ANT & 15 & $12,824,896 \$$ \\
\hline Panvala pan & PAN & 11 & $20,552 \$$ \\
\hline DAOstack & GEN & 9 & $37,553 \$$ \\
\hline Tether USD & USDT & 8 & $1,158,129 \$$ \\
\hline Balancer & BAL & 6 & $331,744 \$$ \\
\hline
\end{tabular}

Table 4: Top 10 currencies by number of DAOs that use them and funds in USD as of 1 st December 2020.

In Table 4, we show the top ten cryptocurrencies in terms of adoption, that is, by the number of DAOs that use them. Ether and Dai are used by 50 and 51 DAOs, respectively, but Ether has more capitalization (close to 15 million dollars versus over 6 million dollars). Regarding the USD capitalization, it is important to bear in mind that the funds of a DAO are dynamic as it has inflows and outflows. The fund data was retrieved the 1st December 2020.

Interestingly, many of those cryptos are stablecoins (DAI, SAI, USDC, or USDT), this means they were designed to keep a stable value during volatile market periods and reduce transaction fees. We can divide them into two groups. The fiatcollateralized type (e.g. USDC, USDT) are the most common stablecoin, and they usually rely on centralized institutions. In the case of crypto-collateralized (e.g. DAI, SAI) coins do not depend on traditional finance infrastructure and use crypto assets as collateral. For example, DAI and SAI cryptos are created by MakerDAO, a non-platform DAO. To get an amount of those cryptos you exchanges ETH to get them [46].

Ether is one of the most used cryptos, despite its market volatility. However, not all DAOs can use ETH as an asset. That is the case of DAOhaus's DAOs, which can not use non-ERC20 cryptocurrencies. Due to that, there are solutions like WETH, that wraps ETH in an ERC20 smart contract. There are other cryptos like ANT or GEN, which are specific tokens for Aragon, and DAOstack platforms. In the case of GEN, it is used for the proposal boosting process. Regarding the ANT token, it is used to govern the Aragon Network DAO. Besides that, each DAO can have its own crypto, for example, PieDAO has DOUGH, a coin with 44,291,262 USD of market capitalization, but it is just owned by PieDAO.

Table 5 has the top ten DAOs with more cryptofunds in USD. Most of those DAOs are from Aragon. Interestingly, most of them have a small number of members (less than 10). For example, mStable is a DAO which provides autonomous and non-custodial stablecoin infrastructure to exchange stablecoins without additional 


\begin{tabular}{|c|c|r|r|}
\hline DAO name & DAO platform & Total cryptocurrencies in USD & Number of members \\
\hline PieDAO & Aragon & $73,829,906 \$$ & 2,881 \\
\hline mStable & Aragon & $38,263,266 \$$ & 8 \\
\hline $\mathrm{dxDAO}$ & DAOstack & $17,581,208 \$$ & 444 \\
\hline Airalab & Aragon & $13,263,696 \$$ & 11 \\
\hline Aragon Trust & Aragon & $7,015,477 \$$ & 5 \\
\hline Aragon Network Budget & Aragon & $5,903,309 \$$ & 3 \\
\hline MetaCartel Ventures & DAOhaus & $5,619,718 \$$ & 99 \\
\hline Aavegotchi & Aragon & $5,059,662 \$$ & 3 \\
\hline API3 DAOv1 & Aragon & $2,991,833 \$$ & 30 \\
\hline Aragon Network & Aragon & $2,932,121 \$$ & 5 \\
\hline
\end{tabular}

Table 5: Top 10 DAOs by a total of cryptocurrencies in USD.

fees. ${ }^{[16]}$ PieDAO is focused on bringing market accessibility and economic empowerment, it allocates DAO resources by governance, in order to gain profit for its investments. ${ }^{[17]}$ In the case of $d x D A O$, it is a DAOstack DAO that obtains revenues from its DeFi services or products they have and/or develop. ${ }^{[18]}$ Finally, MetaCartel Ventures is a DAOhaus DAO for-profit created for investing into early-stage Decentralized Applications or DApps. ${ }^{[19]}$

\section{Discussion}

We have compared the three main DAO ecosystems using four dimensions: growth, activity, voting statistics, and funds. According to the numbers, Aragon is the biggest and most active platform. Moreover, eight of the top ten richest DAOs are from Aragon. Regarding DAOhaus, it is growing in recent times. The voting system seems to be the easiest one to approve a proposal. Regarding DAOstack, the numbers of activity and adoption show signs of stagnation, even after the adoption of $x$ Dai. Still some communities remain loyal and active including one of the most numerous and wealthiest ones, which is $d x D A O$.

Due to the surges in the gas price, the platforms offered to possibility to operate in the $x D a i$ network. However, our numbers do not show a strong effect on the platform's activity, as we expected. In any case, Ethereum 2.0 is expected to finish or at least to mitigate the problems of gas cost, so this xDai alternatives could be temporary solutions.

In general, we have to remark that the conclusions drawn from the statistics retrieved have to be taken with caution. First, because we are looking at general statistics (counts, rates and general trends) without looking into the individual communities. Given the small number of DAO and the potential diversity of DAOs, these figures could be misleading. Second, because the figures reflect the activity of the early-adopters interacting with a new technology and in some cases, some of them may have already abandon the technology or are using it for testing their capabilities.

Despite these shortcomings, we believe it is necessary to advance in the understanding of this new form of online organization that it is taking shape in the blockchain and that is implementing innovative forms of governance. The people

\footnotetext{
${ }^{[16]}$ https://docs.mstable.org/

${ }^{[17]}$ https: //docs . piedao.org/

${ }^{[18]}$ https://dxdao.eth.link/\#/faq

${ }^{[19]}$ https : //metacartel. xyz/about
} 
that design DAO mechanisms usually do it without extensive testing and, as a result, these first organizations can be seen as guinea pigs that are experimenting with a novel system for the first time, while at the same time being the object of the experiment. Furthermore, these new organizations are strongly influenced by the underlying technology, a costly, immutable, decentralized and transparent database, and hence their collective behavior could be different to organizations that operate through standard client-server applications deployed on the Internet.

All these aspects make DAOs a challenging research field. We particularly consider important to dive into the voting systems and how they are affected by aspects such as funds, reputation, etc. We hope our article stimulates the research on these novel communities to help them to deliver truly effective decentralized and scalable governance.

\section{Abbreviations}

- Dapp: Decentralized application.

- DAO: Decentralized Autonomous Organizations.

- DeFi: Decentralized Finance.

- CV: Conviction Voting.

- HC: Holographic Consensus.

\section{Availability of data and materials}

The datasets generated and/or analysed during the current study are available in the following GitHub repository, https://github.com/Grasia/DAO-comparison-data.

Many of that data was extracted from DAO-Analyzer available in the following GitHub repository,

https://github.com/Grasia/dao-analyzer, and deployed in http://dao-analyzer.science/.

The cryptocurrency data used was extracted from the DeepDAO web-tool, http://deepdao.world.

Competing interests

The authors declare that they have no competing interests.

Funding

This work was partially supported by the project P2P Models (https://p2pmodels.eu) funded by the European Research Council (ERC-2017-STG 625 grant no.: 759207), and by the project Chain Community (grant no.: RTI2018-096820-A-100) funded by the Spanish Ministry of Science and Innovation.

Authors' contributions

YFR carried out the research, retrieving and analyzing the data metrics for the DAO comparison and implementing those metrics in DAO-Analyzer. He also wrote the body of the manuscript. JA planned and supervised the research, analyzed the data and co-wrote the introduction, the quantitative comparison and the discussion section. SH planned the research and co-wrote the introduction, the blockchain and DAO sections and the discussion.

Acknowledgements

Not applicable

Author details

${ }^{1}$ Dpt. Software Engineering and Artificial Intelligence, Universidad Complutense de Madrid, Spain. ${ }^{2}$ Institute of Knowledge Technology, Universidad Complutense de Madrid, Spain. ${ }^{3}$ Berkman Klein Center, Harvard University, USA.

References

1. Filippi, P.D., Hassan, S.: Blockchain technology as a regulatory technology: From code is law to law is code. First Monday 21(12) (2016). doi:10.5210/fm.v21i12.7113

2. Zheng, Z., Xie, S., Dai, H.-N., Chen, X., Wang, H.: Blockchain challenges and opportunities: A survey. International Journal of Web and Grid Services 14(4), 352-375 (2018)

3. Rozas, D., Tenorio-Fornés, A., Díaz-Molina, S., Hassan, S.: When Ostrom Meets Blockchain: Exploring the Potentials of Blockchain for Commons Governance. Available at SSRN 3272329 (2018)

4. Ziolkowski, R., Miscione, G., Schwabe, G.: In: ICIS 2020 Proceedings. Blockchain, DLT and Fintech (2020)

5. El Faqir, Y., Arroyo, J., Hassan, S.: An overview of decentralized autonomous organizations on the blockchain. In: Proceedings of the 16th International Symposium on Open Collaboration. Association for Computing Machinery, New York, NY, USA (2020). doi:10.1145/3412569.3412579

6. Valiente, M.-C., Hassan, S., Pavón, J.: Evaluating the Software Frameworks for Developing Decentralized Autonomous Organizations (2020). https://eprints.ucm.es/id/eprint/62240/ 
7. Underwood, S.: Blockchain beyond bitcoin. ACM New York, NY, USA (2016)

8. Hofmann, F., Wurster, S., Ron, E., Böhmecke-Schwafert, M.: The immutability concept of blockchains and benefits of early standardization. In: 2017 ITU Kaleidoscope: Challenges for a Data-Driven Society (ITU K), pp. 1-8 (2017). IEEE

9. Nakamoto, S., Bitcoin, A.: A peer-to-peer electronic cash system. Bitcoin.-URL: https://bitcoin. org/bitcoin. pdf (2008)

10. Hu, Y., Valera, H.G.A., Oxley, L.: Market efficiency of the top market-cap cryptocurrencies: Further evidence from a panel framework. Finance Research Letters 31, 138-145 (2019)

11. Wood, G., et al:: Ethereum: A secure decentralised generalised transaction ledger. Ethereum project yellow paper 151(2014), 1-32 (2014)

12. Dannen, C.: Introducing Ethereum and Solidity vol. 1. Springer, ??? (2017)

13. Vujičić, D., Jagodić, D., Randić, S.: Blockchain technology, bitcoin, and Ethereum: A brief overview. In: 2018 17th International Symposium INFOTEH-JAHORINA, pp. 1-6 (2018). IEEE

14. Casino, F., Dasaklis, T.K., Patsakis, C.: A systematic literature review of blockchain-based applications: current status, classification and open issues. Telematics and Informatics 36, 55-81 (2019)

15. Metcalfe, W.: Ethereum, Smart Contracts, DApps. In: Blockchain and Crypt Currency, pp. 77-93. Springer, ??? (2020)

16. Cocco, L., Pinna, A., Marchesi, M.: Banking on Blockchain: Costs Savings Thanks to the Blockchain Technology. Future Internet 9, 25 (2017). doi:10.3390/fi9030025

17. Cawrey, D.: 37Coins Plans Worldwide Bitcoin Access with SMS-Based Wallet (2014). https://www . coindesk. com/37coins-plans-worldwide-bitcoin-access-sms-based-wallet

18. Liu, B., Szalachowski, P.: A first look into defi oracles. arXiv preprint arXiv:2005.04377 (2020)

19. Christidis, K., Devetsikiotis, M.: Blockchains and Smart Contracts for the Internet of Things. IEEE Access 4 , 2292-2303 (2016)

20. Hassan, S., De Filippi, P.: Decentralized autonomous organizations (glossary of distributed technologies). Internet Policy Review (2021)

21. Wang, S., Ding, W., Li, J., Yuan, Y., Ouyang, L., Wang, F.: Decentralized autonomous organizations: Concept, model, and applications. IEEE Transactions on Computational Social Systems 6(5), 870-878 (2019). doi:10.1109/TCSS.2019.2938190

22. Buterin, V., et al.: A next-generation smart contract and decentralized application platform. white paper 3(37) (2014)

23. Buterin, V.: DAOs, DACs, DAs and More: An Incomplete Terminology Guide (2014)

https://blog. ethereum.org/2014/05/06/daos-dacs-das-and-more-an-incomplete-terminology-guide/

24. Hsieh, Y.-Y., Vergne, J.-P., Anderson, P., Lakhani, K., Reitzig, M.: Bitcoin and the rise of decentralized autonomous organizations. Journal of Organization Design 7(1), 1-16 (2018)

25. Larimer, D.: The Hidden Costs of Bitcoin (2013). https://letstalkbitcoin.com/is-bitcoin-overpaying-for-false-security\#.UjtiUt9xy0w

26. Tikhomirov, S., Voskresenskaya, E., Ivanitskiy, I., Takhaviev, R., Marchenko, E., Alexandrov, Y.: Smartcheck: Static analysis of ethereum smart contracts. In: Proceedings of the 1st International Workshop on Emerging Trends in Software Engineering for Blockchain, pp. 9-16 (2018)

27. Mehar, M.I., Shier, C.L., Giambattista, A., Gong, E., Fletcher, G., Sanayhie, R., Kim, H.M., Laskowski, M.: Understanding a revolutionary and flawed grand experiment in blockchain: the dao attack. Journal of Cases on Information Technology 21(1), 19-32 (2019)

28. Mannan, M.: Fostering worker cooperatives with blockchain technology: Lessons from the Colony project. Erasmus Law Review 11, 190 (2018)

29. team, A.: The Aragon Manifesto (2018). https://aragon.org/blog/the-aragon-manifesto-4a21212eac03

30. Valiente Blázquez, M.-C., Hassan, S., Pavón Mestras, J.: Results and experiences from developing daos with aragon: A case study (2017)

31. Wang, S., Ding, W., Li, J., Yuan, Y., Ouyang, L., Wang, F.-Y.: Decentralized autonomous organizations: concept, model, and applications. IEEE Transactions on Computational Social Systems 6(5), 870-878 (2019)

32. Santander, A., One, A.: My First Aragon App: Voting supercharged with DAOstack's Holographic Consensus (Part 4) (2019). https://blog.aragon.one/ my-first-aragon-app-voting-supercharged-with-daostacks-holographic-consensus-part-4/

33. Colombo, P.: Committees: an application to delegate certain operations of DAOs (2020). https://medium.com/p2p-models/ committees-an-application-to-delegate-certain-operations-of-daos-to-a-subgroup-of-members-1e16703f0af 4

34. Colombo, P.: How does Committees work? (2020) https://medium.com/p2p-models/how-does-committees-work-d7ed116a7306

35. Zargham, M.: Sensor Fusion and Social Choice. https://github.com/BlockScience/conviction/blob/master/social-sensorfusion.pdf (2018)

36. Emmett, J.: Conviction Voting: A Novel Continuous Decision Making Alternative to Governance. https://medium.com/giveth/ conviction-voting-a-novel-continuous-decision-making-alternative-to-governance-aa $746 \mathrm{cfb} 9475$ (2019)

37. Mathematically Formalizing the Conviction Voting Algorithm. https://nbviewer.jupyter.org/github/ BlockScience/Aragon_Conviction_Voting/blob/master/algorithm_overview.ipynb

38. Association, A.: Introducing the Conviction Funding community grants pilot. https://aragon.org/blog/introducing-the-conviction-funding-pilot (2020)

39. Field, M.: Decentralized Governance Matters (2018). https://medium.com/daostack/decentralized-governance-first-principles-1fc6eaa492ed 
40. Field, M.: Holographic consensus-part 1 (2018).

https://medium. com/daostack/holographic-consensus-part-1-116a73ba1e1c

41. Field, M.: Holographic Consensus - Part 2.

https://medium.com/daostack/holographic-consensus-part-2-4fd461e8dcde (2019)

42. Faqir-Rhazoui, Y., Arroyo, J., Hassan, S.: A scalable voting system: Validation of holographic consensus in daostack. In: Proceedings of the 54th Hawaii International Conference on System Sciences http://hdl.handle.net/10125/71296, ???, p. 5557 (2021)

43. Soleimani, A., Bhuptani, A., Young, J., Haber, L., Sethuram, R.: The Moloch DAO (2019). https://github.com/MolochVentures/Whitepaper/blob/master/Whitepaper.pdf

44. Turley, C.: Moloch Evolved: V2 Primer.

https://medium.com/raid-guild/moloch-evolved-v2-primer-25c9cdeab455 (2020)

45. Duncan, J.: MolochDAO: a primitive solution.

https://medium.com/metacartel/molochdao-a-primitive-solution-d11cc522b18e (2019)

46. What Is DAl? https://cryptonews.com/coins/dai/ (2019) 
Figures

$\longrightarrow$ DAOhaus (only mainnet) $\multimap$ DAOhaus $\multimap$ Aragon (only mainnet) $\rightarrow$ Aragon

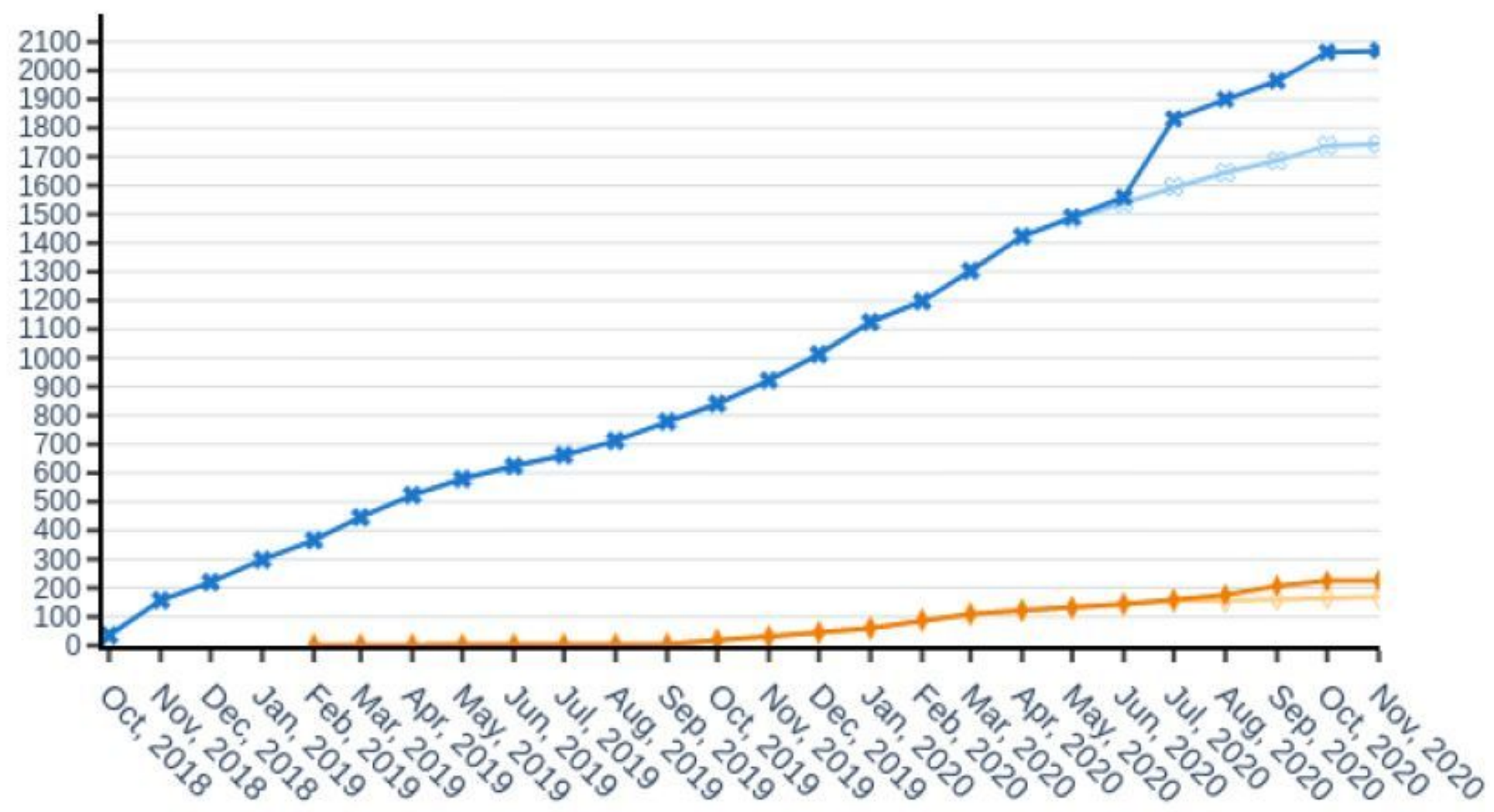

Figure 1

Evolution of the number of DAOs of Aragon and DAOhaus. 
DAOstack (only mainnet) $\longrightarrow$ DAOstack

DAOhaus (only mainnet) $\multimap$ DAOhaus

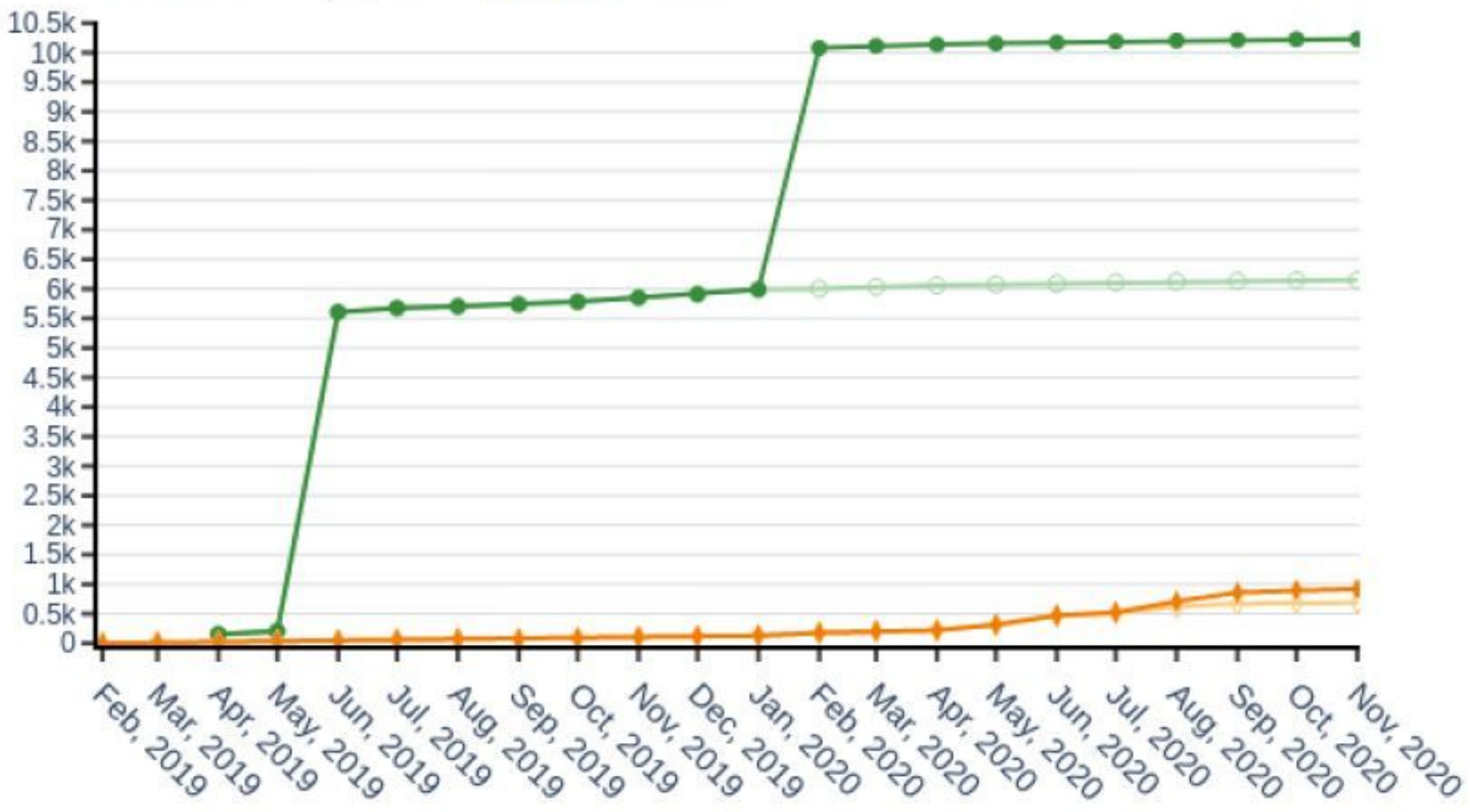

Figure 2

Evolution of the number of users in Aragon and DAOhaus. 


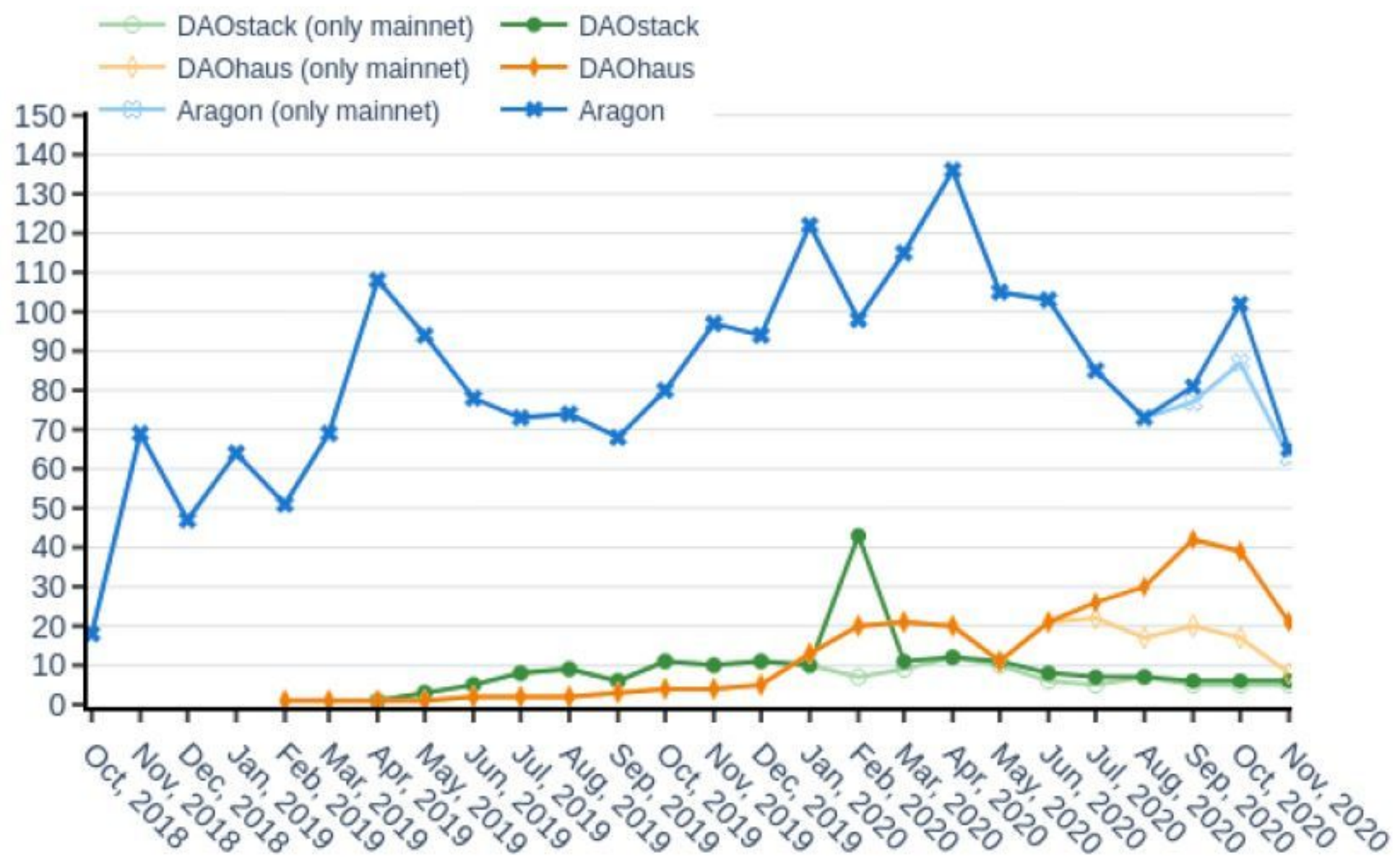

Figure 3

Number of active DAOs in Aragon, DAOhaus and DAOstack. 


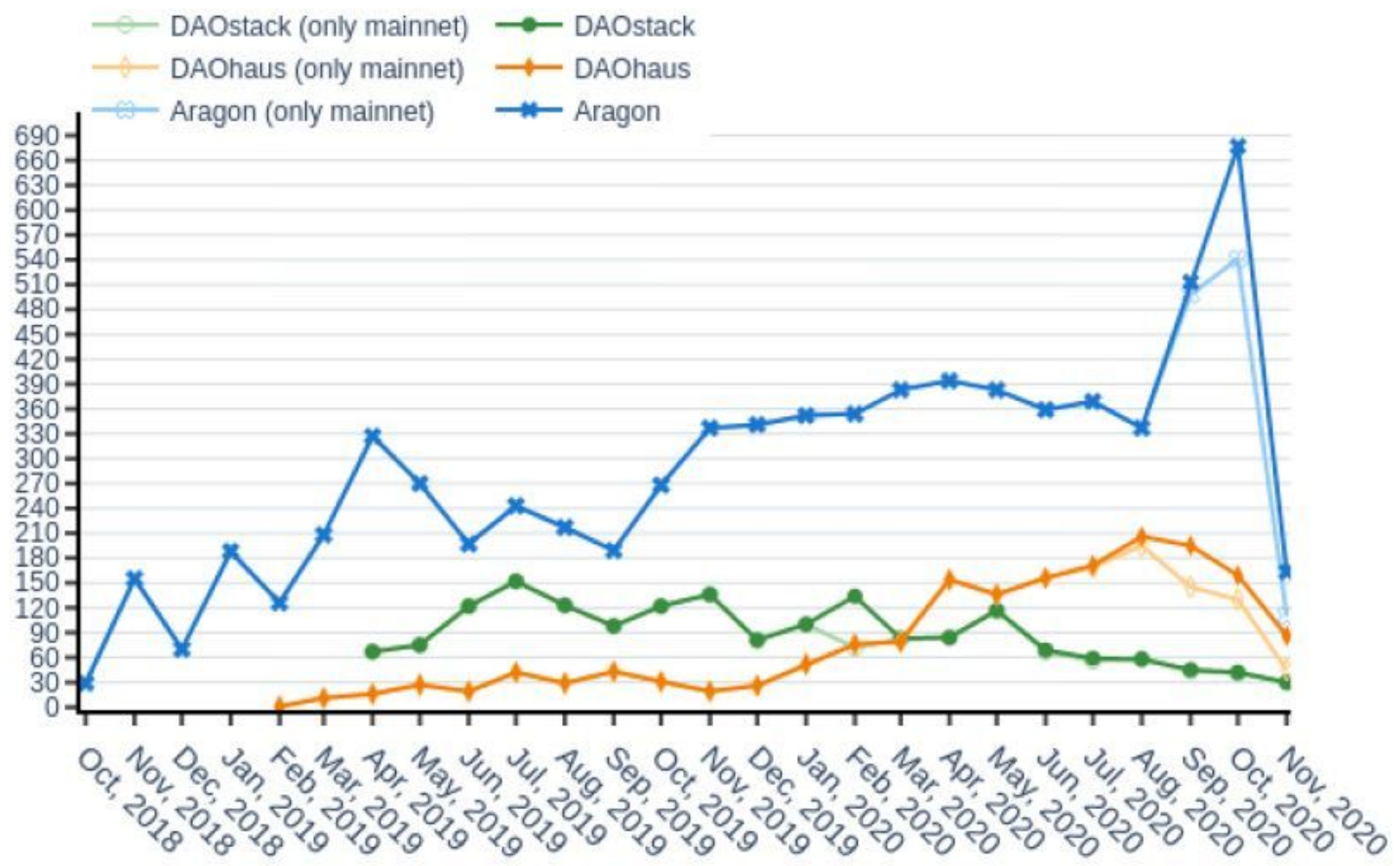

Figure 4

Number of active users of the three platforms. Lighter colors just refer mainnet users. Darker colors take into account users from mainnet and xDai. 